\title{
Pulsatile Tinnitus Related with Prominent Venous Plexus: Case Report and Literature Review
}

\author{
Hwaejoon Jung, Jeong-Rok Kim, Shin Hye Kim, and Jae-Jun Song \\ Department of Otorhinolaryngology-Head and Neck Surgery, Korea University Medical Center, Korea University College of Medicine, \\ Seoul, Korea
}

\author{
돌출된 정맥총에 의한 박동성 이명: 증례 보고와 문헌 고찰 \\ 정회준 · 김정록 · 김신혜 · 송재준 \\ 고려대학교 의과대학 이비인후-두경부외과학교실
}

\author{
Received July 7, 2016 \\ Revised October 4, 2016 \\ Accepted October 13, 2016 \\ Address for correspondence \\ Jae-Jun Song, MD, PhD \\ Department of Otorhinolaryngology- \\ Head and Neck Surgery, \\ Korea University Medical Center, \\ Korea University \\ College of Medicine, \\ 148 Gurodong-ro, Guro-gu, \\ Seoul 08308 , Korea \\ Tel +82-2-2626-1299 \\ Fax $+82-2-868-0475$ \\ E-mail jjsong23@gmail.com
}

Pulsatile tinnitus coexists with patient's heartbeat and objective tinnitus is audible to other persons such as examiner. It can be disagreeable for the patient and can also be the only clue to a potentially devastating and life-threatening disease. Pulsatile tinnitus with a normal otoscopic examination often presents a diagnostic challenge, and its differential diagnosis includes a wide range of conditions. Evaluation of a patient with tinnitus requires a detailed history, a comprehensive audiological evaluation with hearing thresholds, neuro-otological physical examination with otoscopy and imaging studies. We recently experienced a case of a 28 -year-old male complaining of right-sided pulsatile tinnitus. The tinnitus was objectively audible at the left side of the posterior neck. Non-contrast enhanced computed tomography of temporal bone showed a high jugular bulb on the left side. A 6-vessel angiography showed prominent left paravertebral venous plexus and prominent venous plexus around the skull base.

Korean J Otorhinolaryngol-Head Neck Surg 2017;60(1):38-43

Key Words Angiography $\cdot$ Objective tinnitus $\cdot$ Pulsatile tinnitus $\cdot$ Tinnitus.

\section{Introduction}

Tinnitus is a sound in the ear, such as buzzing, ringing, or whistling, occurring without external sound stimulus. ${ }^{1)}$ Approximately $10 \%$ of the population in the USA has tinnitus and $60 \%$ of them have no diagnosis, despite extensive examinations. ${ }^{2)}$ Tinnitus is divided into two types, one perceived only by the patient (subjective) and the other perceptible to another person (objective). ${ }^{1)}$

Pulsatile tinnitus (PT) is synchronized with the patient's heartbeat, whereas non-PT is continuous. Objective tinnitus is audible to the examiner, which is because of a neuromuscular or vascular condition. Objective PT is usually associated with vascular anomalies. According to the vessel of origin, vascular PT can be either arterial or venous. PT due to arterial lesions can be classified into arteriovenous malformation, arteriovenous shunts, intracranial aneurysms, atherosclerosis and fibromuscular dysplasia of the carotid artery. Venous causes of PT include a high or dehiscent jugular bulb, high flow through local emissary veins, local venous stenosis, and idiopathic intracranial hypertension. ${ }^{3)}$

PT with a normal otoscopic examination often poses a diagnostic challenge, and its differential diagnosis includes a wide range of conditions. Various multimodality imaging algorithms have been proposed for its evaluation, which include contrast enhanced computed tomography (CT), magnetic resonance imaging (MRI) with magnetic resonance angiogram (MRA), CT angiography, CT arteriography and venography (CT A/V), and digital subtraction angiography (DSA). ${ }^{4}$

We report a rare case with prominent venous plexus of the 
paravertebral and upper cervical, presenting PT with literature review.

\section{Case}

A 28-year-old male presented with a 2-month history of worsening right-sided PT. He had no history of otitis media, vertigo, otorrhea, or hearing impairment. He didn't complain of headache and visual disturbance. His medical history was otherwise unremarkable. His otoscopic finding was normal (Fig. 1A and B). Pure tone audiometry was performed. Both air and bone conduction hearing levels were measured using the 3 -frequency method in 500, 1000, and $2000 \mathrm{~Hz}$. And the audiometric testing showed normal hearing (right $5 / 5 \mathrm{~dB}$ HL, left 2/2 dB HL) (Fig. 1C).

The tinnitus was auscultated objectively at left side of the posterior neck and audible louder when his head was flexed and turned to the right side (Fig. 2A and B). But the tinnitus disappeared after his head was turned to the left side. There was no change during inspiration, expiration, neck compression and Valsalva maneuver. This sound was synchronous with his heartbeat. We recorded his tinnitus using a portable
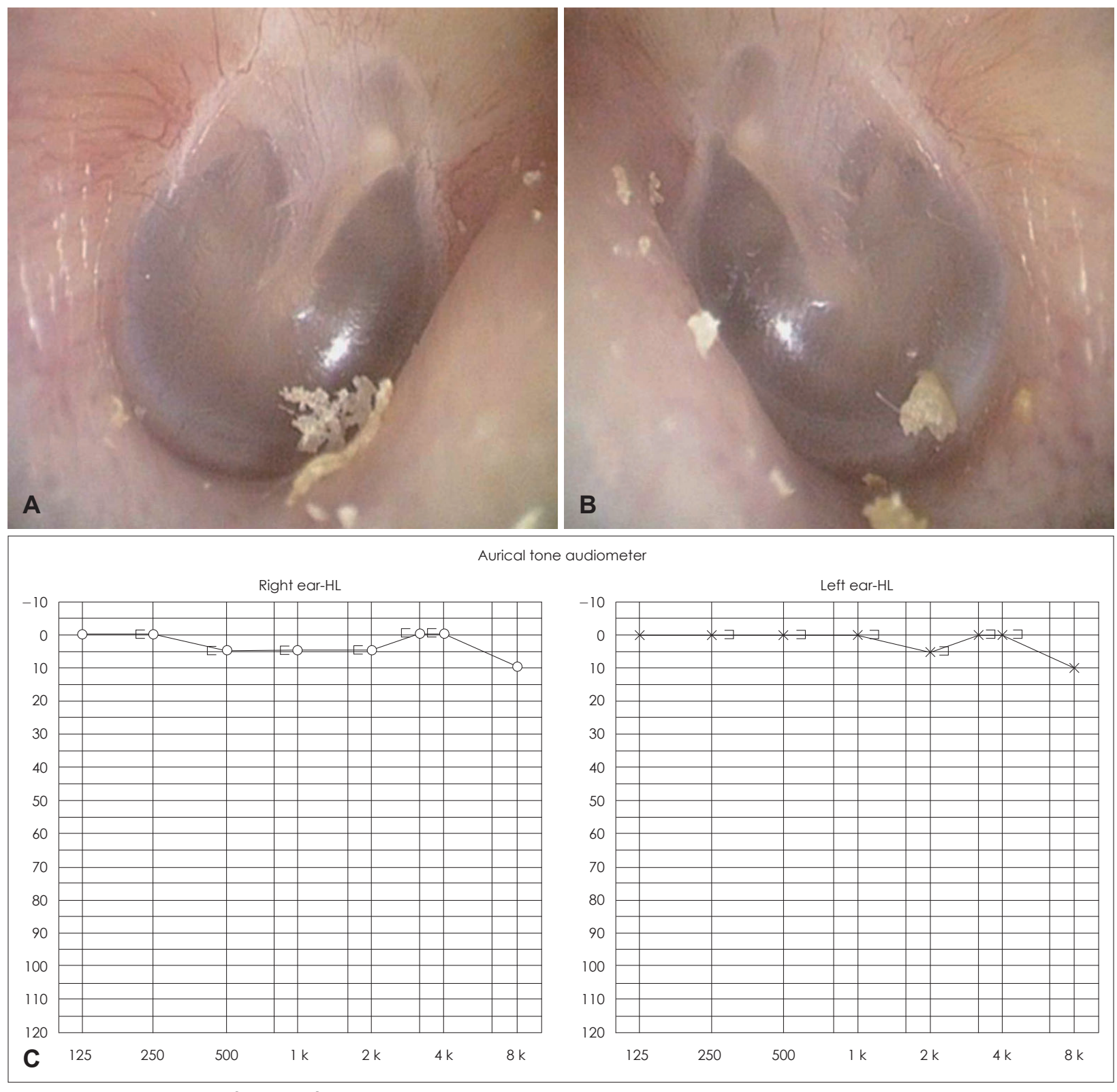

Fig. 1. The otoendoscopic findings of both tympanic membranes. Both tympanic membranes had normal appearance (A and B). The result of pure tone audiometry (C). 
Doppler sonograph and the soundwave showed blood flow synchronous with heartbeat (Fig. 2C).

Non-enhanced CT of temporal bone showed a high jugular bulb on the left side (Fig. 3). There was no bony dehiscence at both jugular bulbs. MRI supplemented with MRA was performed. MRA showed abnormal vascular structure in the left side of the posterior upper neck and prominent paravertebral venous plexus (Fig. 4). Angiography was performed to rule out dural arteriovenous fistula. In 6-vessel angiography, prominent left paravertebral venous plexus and prominent venous plexus around the skull base at the level of hypoglossal canal were seen, but there was no direct evidence of a definite vas-
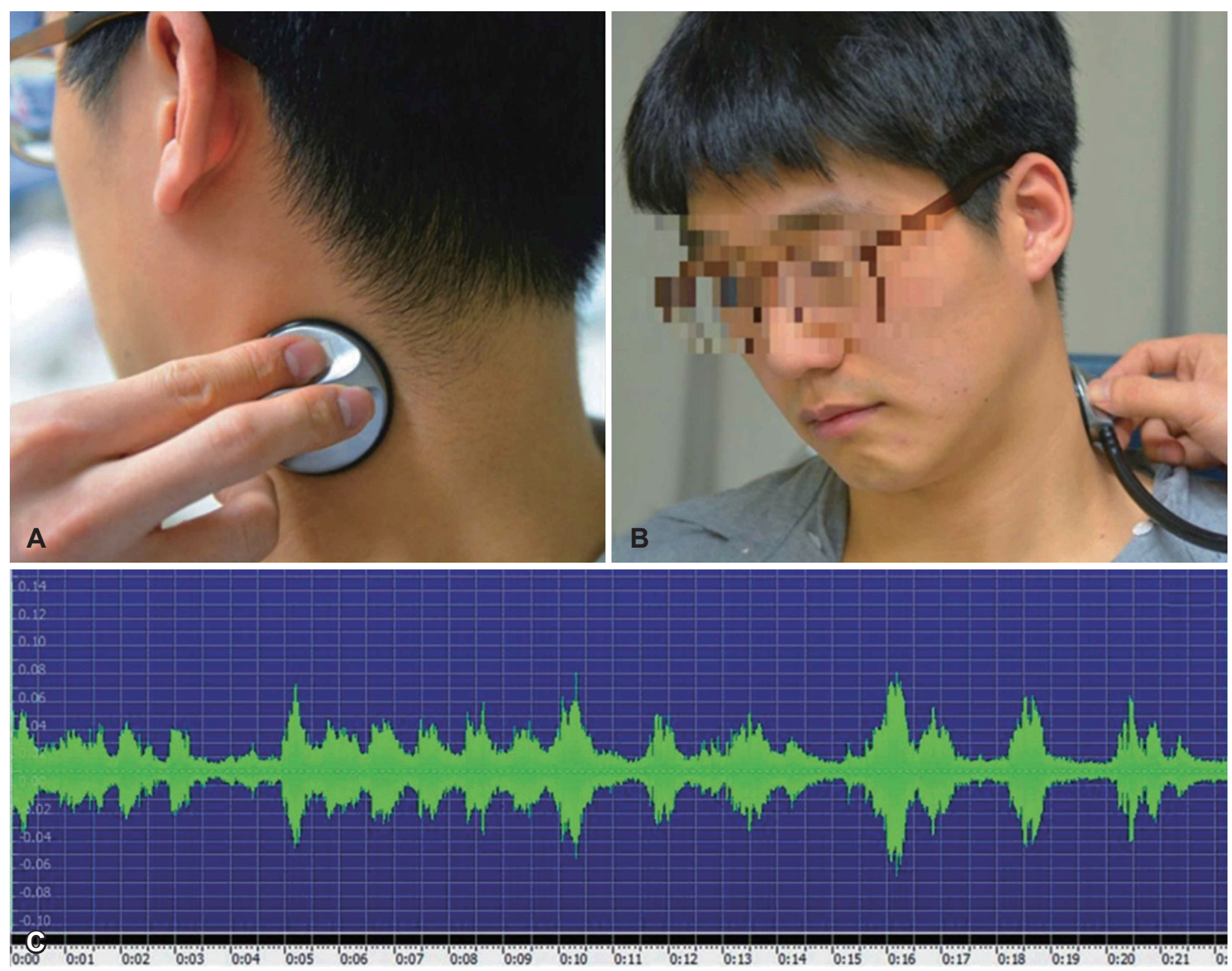

Fig. 2. The position of auscultation with a stethoscope in the outpatient clinic. The patient presented tinnitus that was auscultated objectively at his left posterior neck (A). The tinnitus was audible louder when he turned his head to right and flexed his head (B). Recording the tinnitus sound: the pulsatile sound is synchronous with the heartbeat [x-axis: time (sec), y-axis: amplitude] (C).

Fig. 3. The coronal view of non-enhanced CT of temporal bone showed left high jugular bulb (arrow). Right side (A). Left side (B).
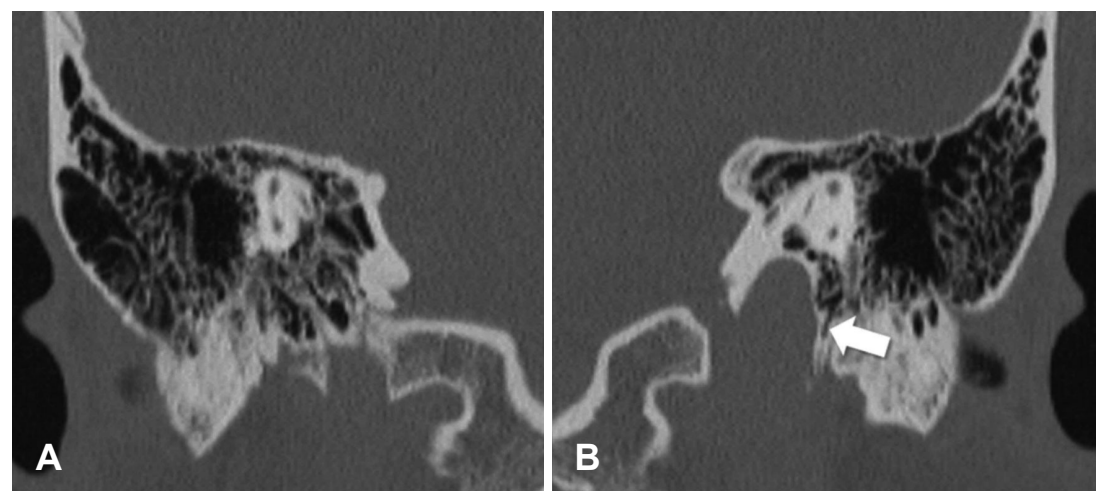
cular abnormality such as arteriovenous fistula (Fig. 5).

Venous lesion found in both MRA and angiography was found to be an anatomical variant; the patient had no cardiovascular complication or symptoms except prolonged PT. Results from imaging modalities were notified to the patient, yet any medical treatments or surgical interventions were refused. Respecting the patient's decision, regular outpatient clinic fol-

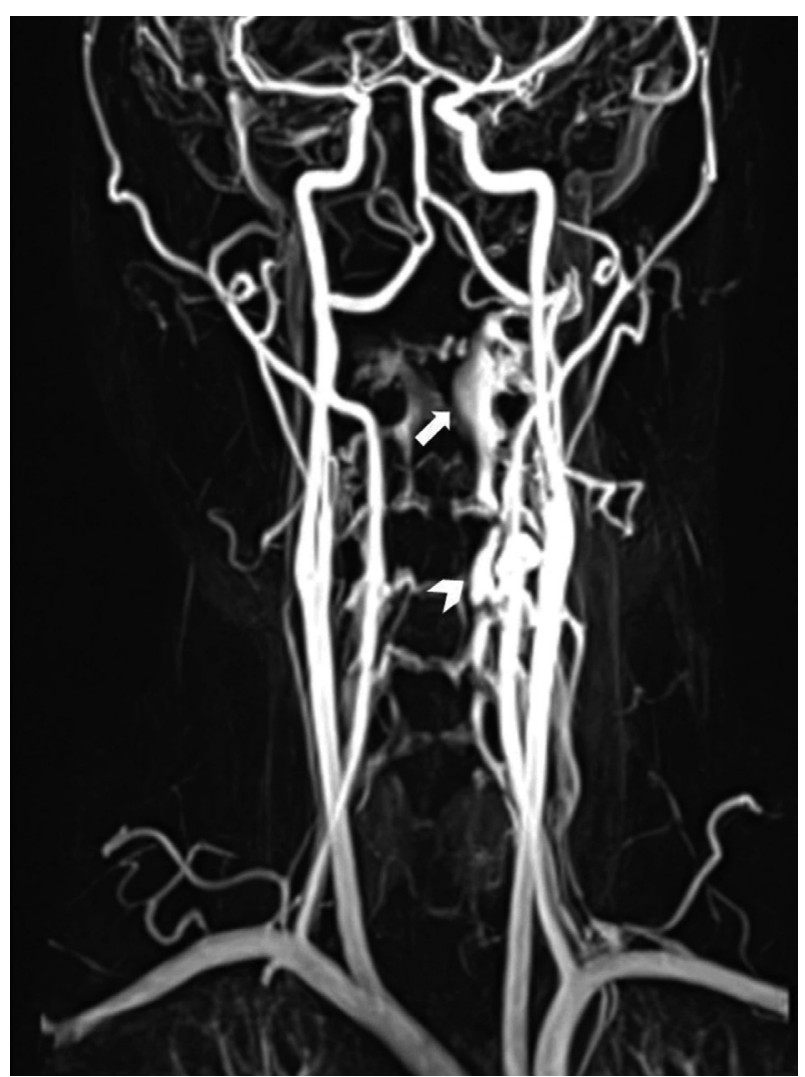

Fig. 4. The findings of MRA of brain: abnormal vascular structure was shown in the left posterior upper neck (arrow) and prominent paravertebral venous plexus (arrowhead). MRA: magnetic resonance angiogram. low-up was scheduled. 7 months have passed since the admission; however, no symptomatic change in direction, loudness, and character of tinnitus have been noted.

\section{Discussion}

Objective tinnitus is when the noise is heard by both the patient and the examiner. ${ }^{2)}$ Objective tinnitus can be further divided into pulsatile and non-pulsatile; PT suggests the presence of vascular malformations, vascular tumors, and congenital or acquired vasculopathies. ${ }^{1)}$ The source of the PT is turbulent blood flow that may occur in the internal jugular vein (IJV) or carotid artery transmitted to the adjacent inner ear through a thin bone plate, or by direct contact of the jugular wall with the semicircular canal. ${ }^{5)}$

The prevalence of PT with venous origin is more common than that of arterial origin. Mattox and Hudgins ${ }^{6}$ reported 54 cases of PT and it showed venous (43\%) origin was more prevalent than arterial $(26 \%)$ and indeterminate $(28 \%)$ origin. In approximately $70 \%$ of patients with PT, the diagnosis will be achieved by imaging studies to make a proper treatment plan. ${ }^{7)}$ Various multimodality imaging algorithms have been proposed for evaluation, which include contrast enhanced CT, MRI with MRA, CT angiography, CT A/V, and DSA. ${ }^{4}$

In our case, MRA and 6-vessel angiography revealed an enlarged venous plexus around the skull base at the level of hypoglossal canal (13 $\mathrm{mm}$ diameter). Other abnormal finding in our patient was prominent paravertebral venous plexus. In general, the anterior condylar confluence is located at the extracranial aperture of the hypoglossal canal at the skull base and, because of its connection to a rich venous network, represents a major venous crossroad. ${ }^{8)}$ It is a constantly observed small venous structure (5 $\mathrm{mm}$ in maximal diameter) of the
Fig. 5. The findings of 6-vessel angiography. Prominent left paravertebral venous plexus (arrow) (A). Prominent venous plexus around the skull base at the level of hypoglossal canal (arrow) (B).
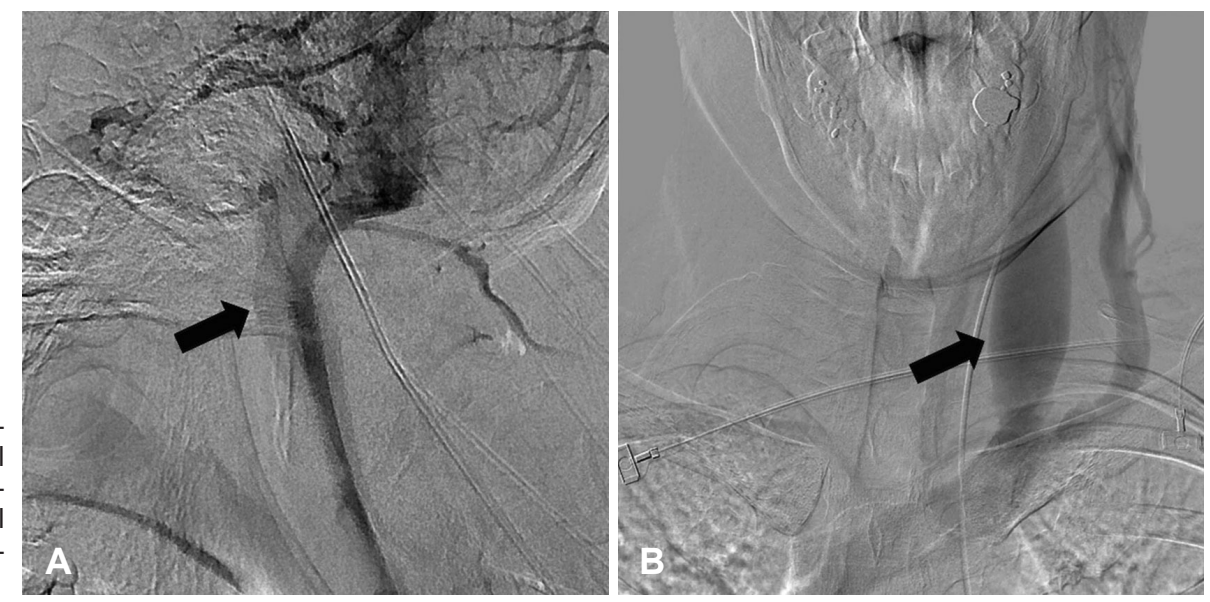
skull base. ${ }^{9)}$

In our patient, left jugular bulb was also found and high as observed in the non-contrast enhanced CT of temporal bone. A high jugular bulb is the most common cause of venous originated tinnitus. A normal jugular bulb is located $2 \mathrm{~mm}$ below the internal acoustic canal. High medial jugular bulb may progressively expand because of centrifugal hemodynamic forces in adult life probably caused by increased cardiac output. Approximately $4.5 \%$ of patients with an enlarged jugular bulb have PT. ${ }^{1)}$

Intracranial hypertension is also known as the cause of venous tinnitus. But in our case, the patient did not complain of headache and visual disturbance, implying intracranial hypertension. Furthermore, the patient did not have any risk factors of intracranial hypertension, which include female sex, middle-aged, menstrual irregularities, obesity, drug and hormonal change. $^{10)}$

There are several unusual findings in this case. First of all, the patient showed right sided PT, but prominent venous plexus was found on the left side. There were no other vascular abnormalities except prominent left paravertebral venous plexus and venous plexus around the skull base at the level of hypoglossal canal. In general, venous tinnitus is frequently perceived at the right side than the left side, because the right jugular vein is usually dominant in $70-80 \%$ of patients. ${ }^{11)}$ The dominant venous system is considered to be a potential factor of the PT. Wang, et al. ${ }^{12)}$ showed $57.41 \%$ of cases with unilateral PT where patients were presented with ipsilateral dominance of the venous system but $9.26 \%$ of patients had contralateral venous dominance. The mechanism is still challenging that explains objective PT on the contralateral side of the venous dominant side.

Secondly, physical examinations did not correspond with clinical findings of usual venous tinnitus. PT disappeared when the patient's head was turned to the left side, but there was no change during compression of the patient's left neck. Light digital compression of the ipsilateral neck and full head rotation toward the side of the tinnitus result in a decrease or complete cessation of venous tinnitus. And venous tinnitus worsened with Valsalva maneuver or rotation of head to opposite side of the tinnitus. ${ }^{1,5)}$ In this case, venous abnormalities were found at left paravertebral venous plexus and at the level of hypoglossal canal. Since these venous systems are located deep within the neck, they seemed not to be compressed sufficiently by manual compression and head rotation.

In PT with venous origin, a variety of treatments have been proposed which aim at interrupting the flow through the IJV, or correcting the venous malformation either through an open surgical procedure or endovascular intervention. Berguer and Nowak ${ }^{5)}$ reported successful results of IJV ligation below facial vein. However, IJV occlusion has been reported to provoke intracranial hypertension, superior vena cava syndrome, cerebral venous ischemia and even pulmonary embolism. Recent reports described the exclusion of sinus diverticula either by endovascular coiling alone, or in association with stenting. ${ }^{13-15)}$ Sanchez, et al. ${ }^{15)}$ demonstrated that the use of the stent with coils in patients with PT can significantly increase the safety of the procedure, avoiding coil migration and retaining sinus permeability.

Choosing the appropriate therapy depends on location of the lesion and its relation to surrounding structures, the architecture of the venous system, and the risk of hemorrhage versus thrombosis. ${ }^{14)}$ The gold standard of treatment for PT with venous origin has not been established until now. In our case, the venous lesion seemed to be an anatomical variant, and the patient had no cardiovascular complications or symptoms, except PT. Considering the patient's preference, we decided regular outpatient clinic follow-up for this variant to check change of tinnitus and relieve the patient's unrest. Endovascular intervention using coiling and stent should be considered as a therapeutic option if the symptoms worsen or interfere with daily activities.

In summary, we report a case of PT on the right side with prominent venous plexus on the contralateral side. If PT arises due to deep venous lesion, physical examination might not show typical findings. For the adequate diagnosis of a patient with objective tinnitus requires multidirectional evaluation including imaging studies. Further study should be performed to prove the discordance of direction between symptoms and imaging findings in $\mathrm{PT}$.

\section{REFERENCES}

1) Sonmez G, Basekim CC, Ozturk E, Gungor A, Kizilkaya E. Imaging of pulsatile tinnitus: a review of 74 patients. Clin Imaging 2007;31 (2):102-8.

2) Alsherhri H, Alqahtani B, Alqahtani M. Dilated petrosquamosal sinus, mastoid emissary vein, and external jugular vein: a rare cause of pulsatile tinnitus, vertigo, and sensorineural hearing loss. Indian J Otol 2011;17(3):123-6.

3) Santa Maria PL. Sigmoid sinus dehiscence resurfacing as treatment for pulsatile tinnitus. J Laryngol Otol 2013;127 Suppl 2:S57-9.

4) Remley KB, Coit WE, Harnsberger HR, Smoker WR, Jacobs JM, McIff EB. Pulsatile tinnitus and the vascular tympanic membrane: CT, MR, and angiographic findings. Radiology 1990;174(2):383-9.

5) Berguer R, Nowak P. Treatment of venous pulsatile tinnitus in younger women. Ann Vasc Surg 2015;29(4):650-3. 
6) Mattox DE, Hudgins P. Algorithm for evaluation of pulsatile tinnitus. Acta Otolaryngol 2008;128(4):427-31.

7) Marsot-Dupuch K. Pulsatile and nonpulsatile tinnitus: a systemic approach. Semin Ultrasound CT MR 2001;22(3):250-70.

8) San Millán Ruíz D, Gailloud P, Rüfenacht DA, Delavelle J, Henry F, Fasel JH. The craniocervical venous system in relation to cerebral venous drainage. AJNR Am J Neuroradiol 2002;23(9):1500-8.

9) Spittau B, Millán DS, El-Sherifi S, Hader C, Singh TP, Motschall E, et al. Dural arteriovenous fistulas of the hypoglossal canal: systematic review on imaging anatomy, clinical findings, and endovascular management. J Neurosurg 2015;122(4):883-903.

10) Markey KA, Mollan SP, Jensen RH, Sinclair AJ. Understanding idiopathic intracranial hypertension: mechanisms, management, and future directions. Lancet Neurol 2016;15(1):78-91.

11) Friedmann DR, Eubig J, McGill M, Babb JS, Pramanik BK, Lalwani AK. Development of the jugular bulb: a radiologic study. Otol
Neurotol 2011;32(8):1389-95.

12) Wang GP, Zeng R, Liu ZH, Liang XH, Xian JF, Wang ZC, et al. Clinical characteristics of pulsatile tinnitus caused by sigmoid sinus diverticulum and wall dehiscence: a study of 54 patients. Acta Otolaryngol 2014;134(1):7-13.

13) Signorelli F, Mahla K, Turjman F. Endovascular treatment of two concomitant causes of pulsatile tinnitus: sigmoid sinus stenosis and ipsilateral jugular bulb diverticulum. Case report and literature review. Acta Neurochir (Wien) 2012;154(1):89-92.

14) Mehanna R, Shaltoni H, Morsi H, Mawad M. Endovascular treatment of sigmoid sinus aneurysm presenting as devastating pulsatile tinnitus. A case report and review of literature. Interv Neuroradiol 2010;16(4): 451-4.

15) Sanchez TG, Murao M, de Medeiros IR, Kii M, Bento RF, Caldas $\mathrm{JG}$, et al. A new therapeutic procedure for treatment of objective venous pulsatile tinnitus. Int Tinnitus J 2002;8(1):54-7. 